SLAC-PUB-9564

hep-ph/0211374

November 2002

\title{
Transverse Polarization Signatures of Extra Dimensions at Linear Colliders *
}

\author{
Thomas G. Rizzo \\ Stanford Linear Accelerator Center \\ Stanford University \\ Stanford CA 94309, USA
}

\begin{abstract}
If significant longitudinal polarization of both the electrons and positrons becomes feasible at a future linear collider(LC), it may be possible to use spin rotators to produce transversely polarized beams. Using the transverse polarization of both beams, new azimuthal spin asymmetries can be formed which will be sensitive probes for new physics beyond the Standard Model. Here we demonstrate that these asymmetries are particularly sensitive to the exchange of Kaluza-Klein towers of gravitons, or other spin-2 fields, that are predicted to exist in higher dimensional theories which address the hierarchy problem. These new asymmetries are shown to be able to extend the search reach for such new physics by more than a factor of two, provide an additional tool for isolating the signatures for spin-2 exchange up to mass scales in excess of $10 \sqrt{s}$, and can be used to help differentiate among the proposed solutions to the hierarchy problem below the production threshold for new particles.
\end{abstract}

\footnotetext{
${ }^{*}$ Work supported by the Department of Energy, Contract DE-AC03-76SF00515
} 


\section{Introduction}

New Physics beyond the Standard Model (SM) is expected to lie at or near the TeV scale on rather general grounds. Once this scale is probed by future colliders, such at the LHC and the Linear Collider (LC), this new physics should begin to show itself. What is uncertain is the form this manifestation will take. The most straightforward scenario to visualize and analyze experimentally would be the production of new particles such as SUSY or KaluzaKlein resonances. A second possibility is that new processes which are not allowed within the SM framework will begin to be observed; it may be very difficult in this case to access the underlying theory. Lastly, one can imagine that the data begins show small deviations from the SM predictions for various observables, e.g., cross sections and asymmetries, which grow with increasing energy. This last possibility signals the existence of new physics beyond the kinematic reach of the collider which is manifesting itself in the form of higher dimensional operators, i.e., generalized contact interactions. These operators can arise from the exchanges of new particles, too massive to be directly produced, with different spins and in various channels depending upon the particular model. From the literature it is easy to construct a rather long list of potential new physics scenarios of this type; clearly this list does not exhaust all of the possibilities: a $Z^{\prime}$ from an extended gauge model[1, 2], scalar or vector leptoquarks[1, 3], $R$-parity violating sneutrino( $\tilde{\nu})$ exchange[4], scalar or vector bileptons[5], graviton Kaluza-Klein(KK) towers[6, 7] in extra dimensional models[8, 9], gauge boson KK towers[10, 7], and even string excitations[11].

If such deviations are observed it will be necessary to have techniques available to differentiate the multiple possibilities experimentally and point us in the direction of the correct scenario. One possible path to take if this situation is realized is to compare the observed shifts with the predictions of all of the currently available models[12]. An alternative to this approach is to develope specific tools to rapidly identify certain classes of models which 
lead to uniquely distinct signatures. In this paper we examine one such tool which becomes available at the LC provided both the $e^{-}$and $e^{+}$beams are initially longitudinally polarized and spin rotators are used to convert these to transversely polarized beams. As we will see below, transverse polarization (TP)[13] allows for new asymmetries to be constructed which are associated with the azimuthal angle formed by the directions of the $e^{ \pm}$polarization and the plane of the momenta of the outgoing fermions in the $e^{+} e^{-} \rightarrow f \bar{f}$ process. Historically, the possible use of TP as a tool for new physics searches and analyses has not gotten the attention it deserves in the literature[13]. Here, in an effort to partially remedy this situation, we are interested in using the associated TP asymmetries to uniquely probe for the $s$-channel exchange of spin-2 fields in $e^{+} e^{-}$collisions which we normally associate with the KaluzaKlein graviton towers of the Arkani-Hamed, Dimopoulos and Dvali(ADD)[8] or RandallSundrum(RS)[9] scenarios.

The organization of this paper is as follows. After our introduction, we outline the influence of transverse polarization on the process $e^{+} e^{-} \rightarrow f \bar{f}$ in the presence of graviton exchange in both the ADD and RS scenarios. In particular we examine how the new asymmetries associated with transverse polarization can be used to probe for spin-2 graviton-like exchanges. In section 3 we analyze these asymmetries in detail and their applications for spin-2 exchange identification at a future LC. We demonstrate the usefulness of TP in distinguishing $s$-channel spin-2 exchange from other possible new physics scenarios. We will see that TP allows one to expand the sensitivity range for the cutoff scale over which graviton KK exchange can be observed by up to a factor of two. We also show that TP may allow us to differentiate the ADD and RS scenarios below KK production threshold. Our conclusions can be found in Section 4. 


\section{Transverse Polarization Asymmetries}

For our analysis we will follow a slightly modified version of the notations and conventions employed by Hikasa[13]. Consider the process $e^{+} e^{-} \rightarrow f \bar{f}$ with the both electron and positron beams polarized. We will denote the linear and transverse components of the $e^{-}\left(e^{+}\right)$ polarizations by $P_{L, T}\left(P_{L, T}^{\prime}\right)$ and for simplicity assume that the two transverse polarization vectors are parallel up to a sign. In this case, the spin-averaged matrix element for this process can be written as

$$
\begin{aligned}
|\overline{\mathcal{M}}|^{2} & =\frac{1}{4}\left(1-P_{L} P_{L}^{\prime}\right)\left(\left|T_{+}\right|^{2}+\left|T_{-}\right|^{2}\right)+\left(P_{L}-P_{L}^{\prime}\right)\left(\left|T_{+}\right|^{2}-\left|T_{-}\right|^{2}\right) \\
& +\left(2 P_{T} P_{T}^{\prime}\right)\left[\cos 2 \phi \operatorname{Re}\left(T_{+} T_{-}^{*}\right)-\sin 2 \phi \operatorname{Im}\left(T_{+} T_{-}^{*}\right)\right],
\end{aligned}
$$

where $\phi$ is the azimuthal angle defined on an event-by-event basis described above. (Note that $\phi$ is defined in such a way so that the $2 \rightarrow 2$ process always takes place in the $\phi=$

0 plane.) It is interesting to note that the $\phi$-dependent pieces of $|\overline{\mathcal{M}}|^{2}$ are particularly sensitive to the relative phases between the two sets of amplitudes. We also observe from this expression the important fact that the $\phi$-dependent pieces are only accessible if both beams are simultaneously transversely polarized. Thus to have azimuthal asymmetries at a LC we must begin with both beams longitudinally polarized and employ spin rotators.

Let us first consider the simple case with massless fermions in the final state assuming no $s$-channel scalar exchanges are present. Let us define the quantities

$$
\begin{aligned}
f_{L L} & =Q_{e} Q_{f}+g_{Z}\left(v_{e}-a_{e}\right)\left(v_{f}-a_{f}\right) P \\
f_{R R} & =Q_{e} Q_{f}+g_{Z}\left(v_{e}+a_{e}\right)\left(v_{f}+a_{f}\right) P \\
f_{L R} & =Q_{e} Q_{f}+g_{Z}\left(v_{e}-a_{e}\right)\left(v_{f}+a_{f}\right) P \\
f_{R L} & =Q_{e} Q_{f}+g_{Z}\left(v_{e}+a_{e}\right)\left(v_{f}-a_{f}\right) P,
\end{aligned}
$$


where $v_{e}, a_{e}\left(v_{f}, a_{f}\right)$ are the vector and axial vector couplings of the initial electron(final fermion) to the $Z$ and $Q_{e, f}$ are their corresponding electric charges, with

$$
g_{Z}=\frac{G_{F} M_{Z}^{2}}{2 \sqrt{2} \pi \alpha}
$$

and

$$
P=\frac{s}{s-M_{Z}^{2}+i M_{Z} \Gamma_{Z}}
$$

Without scalar exchange but allowing for the possibility of spin-2 the relevant helicity amplitudes for this process are given by

$$
\begin{aligned}
& T_{+-}^{+-}=f_{L L}(1+z)-f_{g}\left(z+2 z^{2}-1\right) \\
& T_{+-}^{-+}=f_{L R}(1-z)-f_{g}\left(z-2 z^{2}+1\right) \\
& T_{-+}^{+-}=f_{R L}(1-z)-f_{g}\left(z-2 z^{2}+1\right) \\
& T_{-+}^{-+}=f_{R R}(1+z)-f_{g}\left(z+2 z^{2}-1\right) .
\end{aligned}
$$

where $z=\cos \theta$. Note that the spin-2 exchange merely augments the amplitudes which are already present in the $\mathrm{SM}$ (though with different $\cos \theta$ dependencies), i.e., no new helicity amplitudes are generated by spin-2 over those due to spin-1. In contrast to this, scalar exchange would yield additional amplitudes of the form $T_{++}^{++}$etc. not present in the SM and would thus be easily isolated using the more conventional asymmetries associated with two beam longitudinal polarization[4]. Clearly isolating spin-2 exchange will be somewhat more difficult since no new amplitudes appear. $f_{g}$ is a model-dependent quantity; in the usual ADD model, employing the convention of Hewett[6], one finds

$$
f_{g}=\frac{\lambda s^{2}}{4 \pi \alpha M_{H}^{4}}
$$


where $M_{H}$ represents the cutoff scale in the KK graviton tower sum and $\lambda= \pm 1$. In the RS model the corresponding expression can be obtained through the replacement

$$
\frac{\lambda}{M_{H}^{4}} \rightarrow \frac{-1}{8 \Lambda_{\pi}^{2}} \sum_{n} \frac{1}{s-m_{n}^{2}+i m_{n} \Gamma_{n}}
$$

where $\Lambda_{\pi}$ is of order a few $\mathrm{TeV}$ and $m_{n}\left(\Gamma_{n}\right)$ are the masses(widths) of the TeV scale graviton KK excitations. In what follows we will always assume that we are below the threshold for the production of these resonances otherwise the spin-2 nature of the new exchange would be easily identified through an examination of the resonances themselves. We will also assume that we are sufficiently distant from these resonances in energy that there widths can be neglected in cross section calculations.

In the case of massive final state fermions, such as tops, the helicity amplitudes given above are slightly altered and new amplitudes $T_{+-}^{ \pm \pm}$and $T_{-+}^{ \pm \pm}$are also present. The exact forms of these expressions in this case are not very enlightening so we will not present them here but they will be included in the analysis in the case of top quark pair production.

What is the form or the angular distribution, $d \sigma / d z d \phi$, in the SM? (Here we define $z=\cos \theta$ as above.) In particular, we are interested in the $z$-dependence of the terms associated with $\cos 2 \phi$ and $\sin 2 \phi$ in the expression above when no new physics is present. We note that the a small 'imaginary' term will be present even in the SM due to the finite width of $Z$. For $\sqrt{s} \geq 500 \mathrm{GeV}$ this term can be safely neglected for most of our analyses here but we include it for completeness. As shown in, e.g., the work of Hikasa, both of these $\phi$-dependent terms are always proportional to $1-z^{2}$ in the SM and will remain so even if new gauge boson exchanges are present. However, due to the more complex $z$-dependence of the spin-2 contributions to the helicity amplitudes we expect significant modifications of the SM result when gravitons are exchanged. In fact, interference between SM and spin-2 exchange amplitudes are found to produce both even and odd $-z$ terms with the latter proportional 
to $\sim z\left(1-z^{2}\right)$ whereas the smaller pure gravity terms are instead found to be even in $z$ and proportional to $z^{2}-\left(2 z^{2}-1\right)^{2}$. The general difference in the $z$-dependence of the of the $\phi$ sensitive terms and, in particular, the existence of the odd- $z$ contributions is clearly a signal for spin-2 exchange. We note in passing that in the case of scalar exchange no odd- $z$ terms will be generated since the spin-0 and spin-1 exchanges do not interfere.

Let us assume, as mentioned above, that we are in an energy regime where the effects of the finite width of the $Z$ can be neglected. For the moment, this would seem to imply that the term proportional to $\sin 2 \phi$ can be neglected in the case of KK graviton exchange. Let us proceed making this assumption but remembering to return to this important point below. We will later see that the terms that we now neglecting will have no influence on this part of our analysis. In order to attempt to isolate the spin-2 exchange contributions we first can form a differential azimuthal asymmetry distribution which we symbolically define by

$$
\frac{1}{N} \frac{d A}{d z}=\left[\frac{\int_{+} \frac{d \sigma}{d z d \phi}-\int_{-} \frac{d \sigma}{d z d \phi}}{\int d \sigma}\right]
$$

where $\int_{ \pm}$are integrations over regions where $\cos 2 \phi$ takes on \pm values; integration over the full ranges of $z$ and $\phi$ occurs in the denominator. It is important to note that we expect this differential asymmetry to take on rather small numerical values since it is normalized to the total cross section and not to the differential cross section at the same value of $z$ as is usually done. As we will see below, this particular normalization is most useful in isolating the most important aspects of TP physiucs. To get a feeling for this asymmetry, we show its behaviour for both the SM and in the ADD scenario in Fig.1 at a $500 \mathrm{GeV}$ LC for the final states $f=\mu$ or $\tau, c$ and $b$. Note that from here on we will combine results for the $f=\mu$ and $\tau$ final states to get added statistics. In this figure we have for concreteness assumed that the spin rotators are nearly $100 \%$ efficient[14] so that $P_{T}=0.8$ and $P_{T}^{\prime}=0.6$. Note that the spin-2 effects are large and in particular the fact that the azimuthal asymmetry distribution 
is no longer symmetric under $z \rightarrow-z$ as we might expect from the discussion above.

There are two ways to naively access the odd- $z$ terms. First, one can take the differential azimuthal asymmetry defined above, separately integrate it over positive and negative values of $z$, then take the difference, i.e., form a forward-backward asymmetry using $N^{-1} d A / d z:$

$$
A_{F B}=\frac{1}{N}\left[\int_{z \geq 0} d z \frac{d A}{d z}-\int_{z \leq 0} d z \frac{d A}{d z}\right] .
$$

It is important to be reminded that in the SM and in any new physics scenario with $s$-channel $Z^{\prime}$ exchanges one has $A_{F B}=0$. This is also true in the usual four-fermion contact interaction scenario[16] which involves only vector and axial-vector couplings. Due to the nature of spin0 exchange it is clear that $A_{F B}$ would remain zero in this case as well. A second possibility is to take odd moments of the asymmetry with respect to, e.g., the Legendre polynomials $P_{n}(z)[15]:$

$$
<P_{n}>=\frac{1}{N}\left[\int d z P_{n}(z) \frac{d A}{d z}\right] .
$$

Note that only $<P_{1,3}>$ will be non-zero in this case since no factors of $z^{5}$ appear in the cross section. As in the case of $A_{F B}$, these moments are zero in both the SM and $Z^{\prime}$ models. In the case of graviton exchange, not only are the moments $\left\langle P_{1,3}>\right.$ non-zero, they are also not independent of each other. A short analysis finds that in the case of spin-2 exchange the ratio of moments is fixed: $\left\langle P_{3}\right\rangle /\left\langle P_{1}\right\rangle=-3 / 7$, uniquely. It is thus rather obvious that the existence of odd- $z$ terms is a signal for graviton, or more generally, spin-2 exchange. 

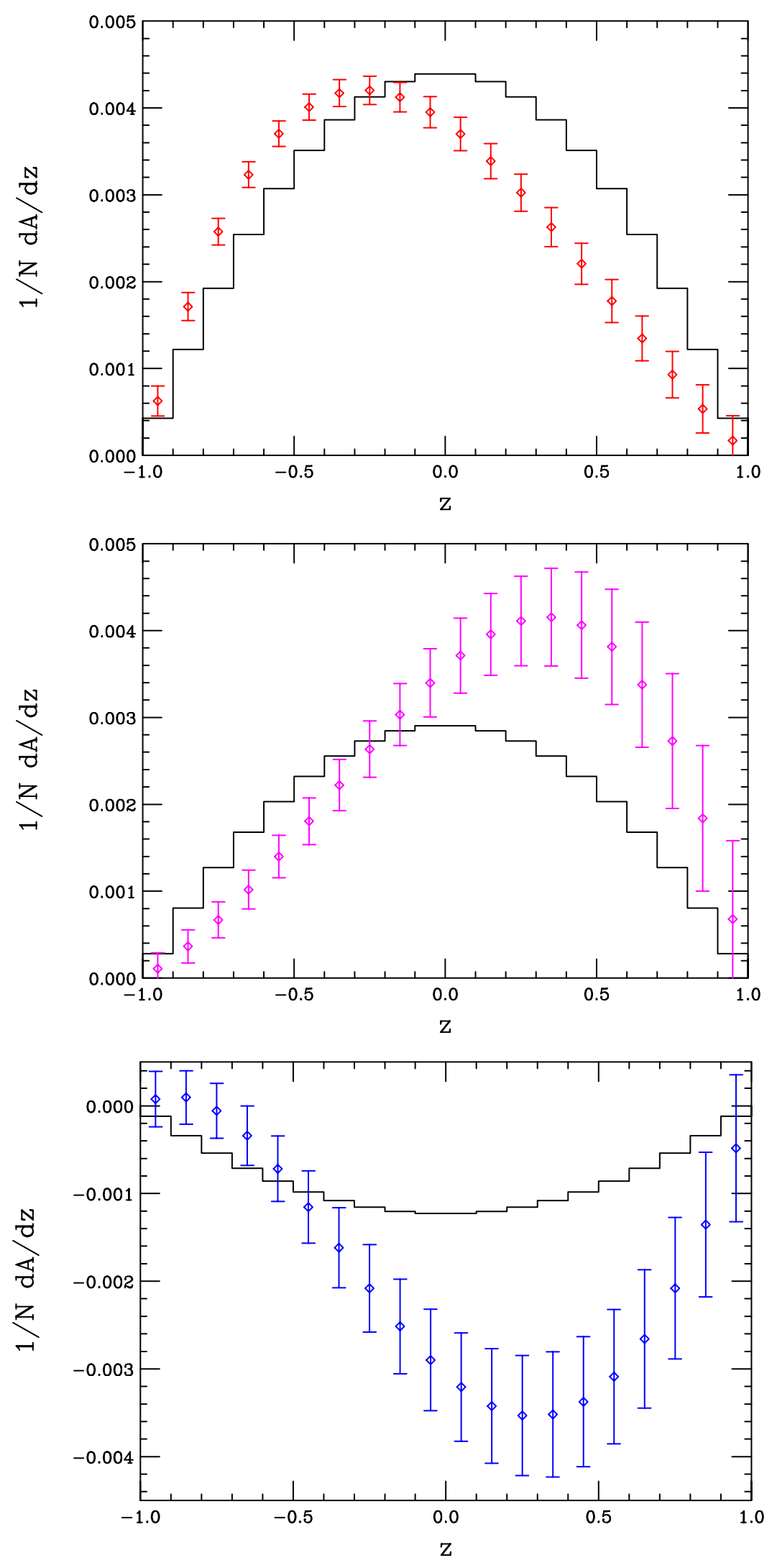

Figure 1: Differential azimuthal asymmetry \&istribution for $e^{+} e^{-} \rightarrow f \bar{f}$ at a $500 \mathrm{GeV} \mathrm{LC}$ assuming a luminosity of $500 \mathrm{fb}^{-1}$. The histograms are the SM predictions while the data points assume the ADD model with $M_{H}=1.5 \mathrm{TeV}$. In the top panel $f=\mu$ and $\tau$ are combined, while in the middle(lower) panel, $f=c(b) . P_{T}=0.8$ and $P_{T}^{\prime}=0.6$ are assumed. 


\section{Analysis}

It is clear that non-zero values of either $A_{F B}$ or $<P_{1,3}>$ provide a clean signature for spin- 2 exchange in the $e^{+} e^{-} \rightarrow f \bar{f}$ process. Their appearance at the level of $5 \sigma$ can thus be claimed as, not just a discovery of new physics, but spin-2 exchange in particular. To be specific in what follows let us concentrate on the ADD model; (almost) all limits obtained there can be immediately translated to the case of the RS scenario. From Fig.1 it is apparent that modest values of $M_{H}$ cause quite sizeable distortions in the $N^{-1} d A / d z$ distribution. However, as we will see this sensitivity is somewhat diluted if we are only asking whether or not, e.g. $A_{F B}$ is non-zero. After all the asymmetry distribution may be quite different than what the SM predicts in both magnitude and shape and yet $A_{F B}$ will remain zero. Such a possibility will occur in the case of , e.g., spin-0 exchange. To determine the $5 \sigma$ identification reach we will assume that the individual polarizations are known rather well, $\delta P / P=0.003$, that the efficiencies of identifying the final state fermions is rather high: $100 \%$ for $f=\mu, \tau, 60 \%$ for $f=c, t$, and $80 \%$ for $f=b$ with no associated systematic uncertainties and include the effects of initial state radiation. The $5 \sigma$ identification reaches, making these assumptions, are shown in Figs. 2 and 3 for different values of $\sqrt{s}$ as functions of the integrated luminosity. In obtaining these results we have combined all of the various final states above into a single fit. In all cases a small angle cut of 100 mrad around the beam pipe has been employed; our results are not particularly sensitive to this value.

These reaches are, as one might expect, somewhat sensitive to the fact that we have assumed values of $P_{T}=0.8$ and $P_{T}^{\prime}=0.6$. If the efficiency of the spin rotators is somewhat less than $\sim 100 \%$ or if such high initial longitudinal polarizations are not achieved the $5 \sigma$ identification reach will clearly degrade. This reduction in reach will not be very

serious unless the product $P_{T} P_{T}^{\prime}$ is very greatly reduced or unless other errors dominate the 


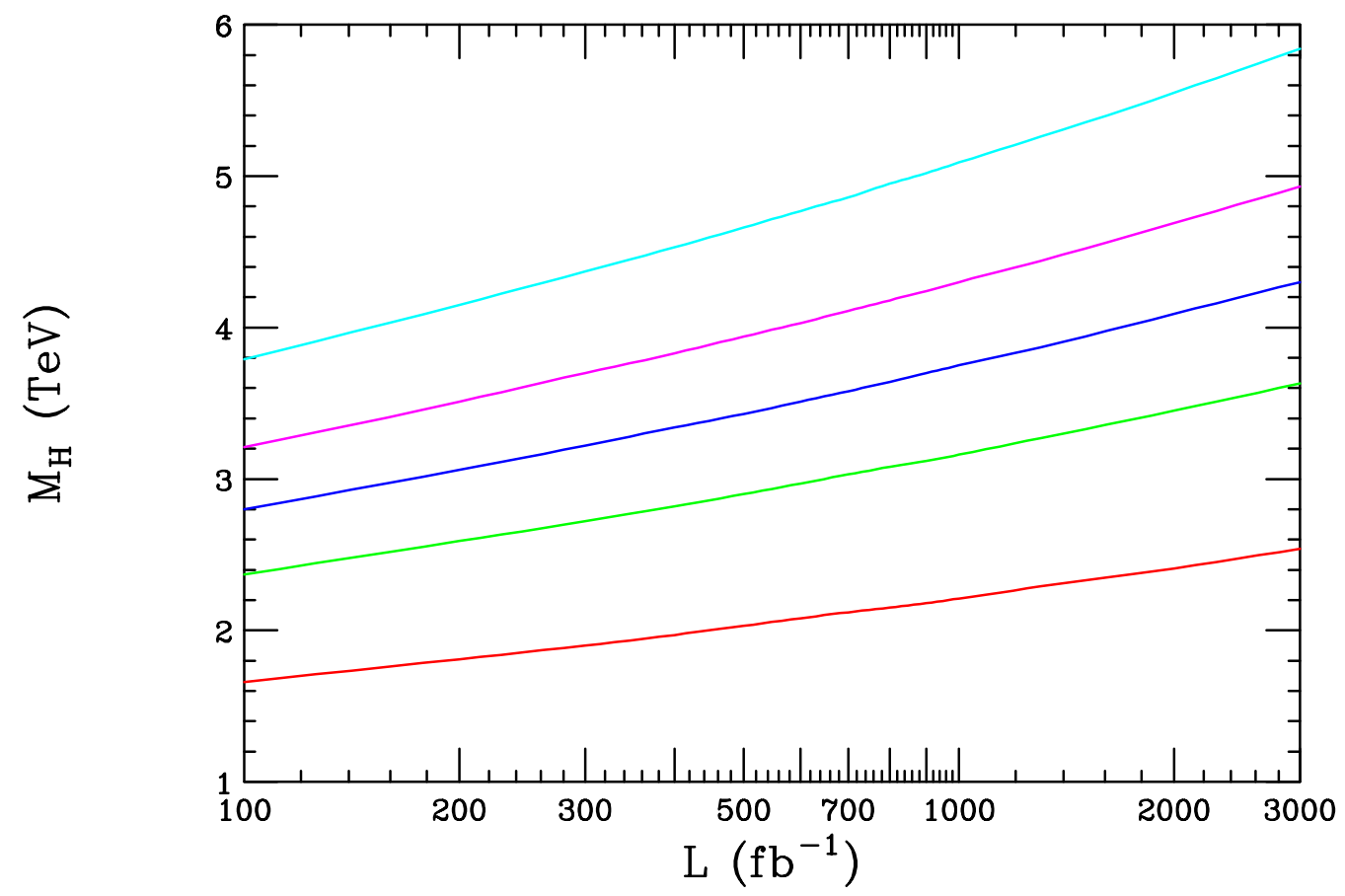

Figure 2: $5 \sigma$ identification reach in $M_{H}$ using $A_{F B}$ as a function of the integrated luminosity from the process $e^{+} e^{-} \rightarrow f \bar{f}$, with $f$ summed over $\mu, \tau, b, c$ and $t$. Here $P_{T}=0.8$ and $P_{T}^{\prime}=0.6$ are assumed. From bottom to top the curves are for $\sqrt{s}=0.5,0.8,1,1.2$ and 1.5 $\mathrm{TeV}$, respectively. 
experimental uncertainties in the measurements.

From these two figures some immediate conclusions can be drawn. First, it is clear that the identification reach obtained from $A_{F B}$ is somewhat superior to that obtained from the measurements of $\left\langle P_{1,3}>\right.$. Secondly, it is clear that the identification reach in either case alone, $M_{H} \sim(3.5-4) \sqrt{s}$, is not as good as what can be obtained employing longitudinal polarization[15]. Thirdly, it is unclear that combining the two sets of observables would be very useful since $A_{F B}$ is correlated with the values of $\left\langle P_{1,3}\right\rangle$. In order to obtain better reaches we must try something more aggressive.

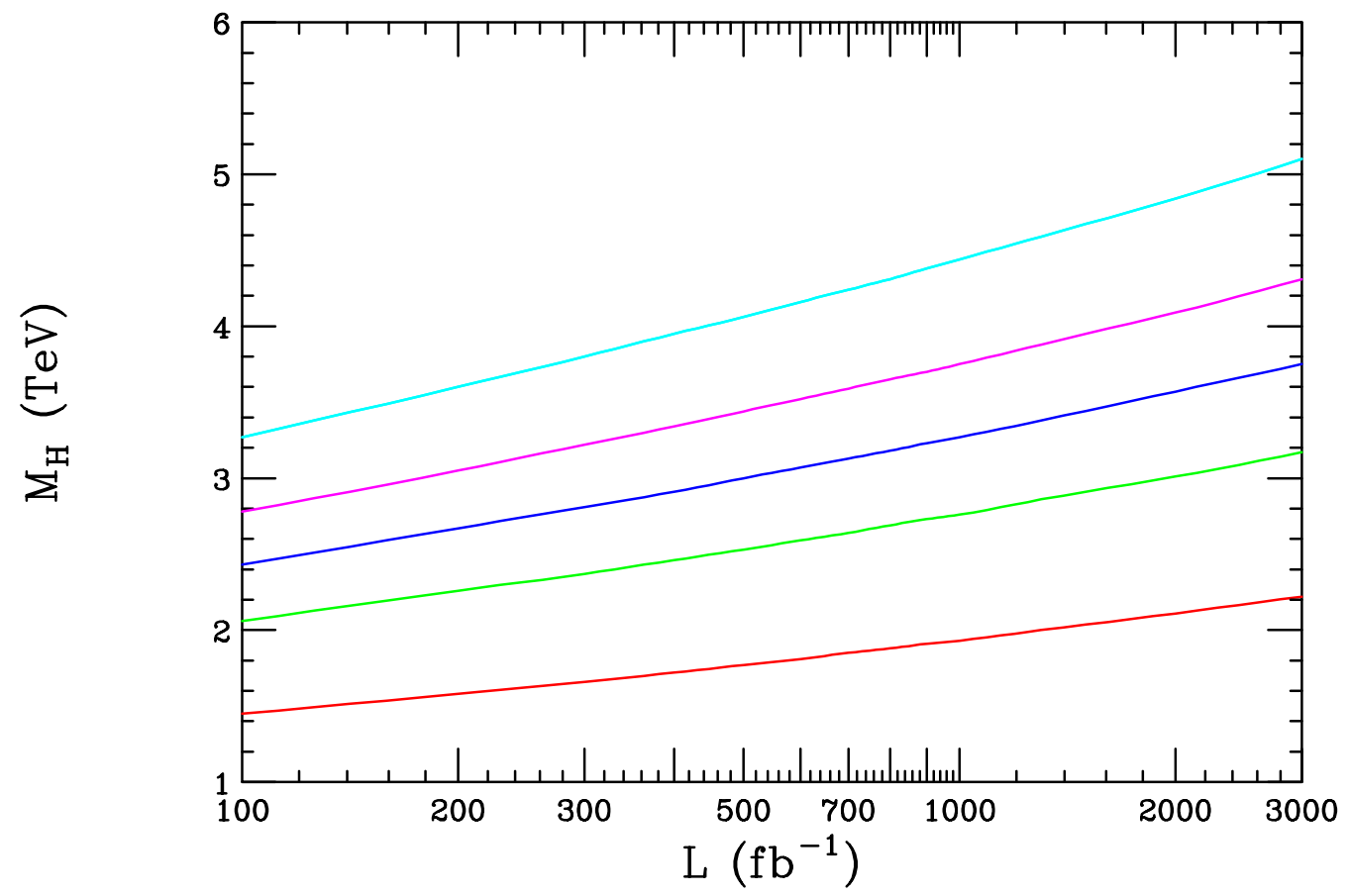

Figure 3: Same as the previous figure but now using the moments $<P_{1,3}>$.

We noted above that in the SM, in all $Z^{\prime}$ models and in the case of conventional fourfermion contact interactions the azimuthal asymmetry always takes the form $N^{-1} d A / d z \sim$ $\left(1-z^{2}\right)$. Clearly these specific forms of new physics will only modify the normalization of the azimuthal asymmetry distribution since its shape is left unaltered. We can thus ask up to what value of the cutoff scale, $M_{H}$, can we differentiate the effects of gravity-a 
change in the shape of these distributions-from a simple overall change in the normalization of distributions for the various final states. This allows us to set a limit on the value of $M_{H}$ below which graviton exchange can be distinguished from $Z^{\prime}$ exchange or four-fermion contact interactions. To do this we fix $M_{H}$ and try to fit the $N^{-1} d A / d z$ distributions for $\mu, \tau, c$ and $b$ final states assuming a SM shape but allowing the normalization to float independently for each final state. If the CL of the fit is very poor we raise $M_{H}$ until we achieve a CL equivalent to $5 \sigma$, i.e., $5.7 \times 10^{-5}$. For luminosities above $100-200 \mathrm{fb}^{-1}$ the errors are completely dominated by systematics and we find the results shown in Table 1. (Changing the luminosities in our range of interest $\frac{1}{2}-2 f b^{-1}$ has little quantitative effect and only modifies the second decimal place in these results.) Here we see that for $M_{H} \lesssim(10-11) \sqrt{s}$ the effects of spin-2 graviton exchange can be distinguished from a $Z^{\prime}$ or any form of the four-fermion contact interactions. This identification reach is numerically similar to the 95\% CL discovery reach for graviton exchange obtained using only singly longitudinally polarized beams $[15,17,18]$ for the same process. Since these results are so dominated by the systematics it is important that a more detailed study of this type be performed using a realistic detector simulation since the likely size of the true systematic errors will be somewhat larger than those assumed in this analysis. However, the reach here is so large it is clear that this is an avenue worth persuing.

Given these results we can go a step further. If graviton and $Z^{\prime}$ exchanges can be distinguished up to $M_{H} \lesssim(10-11) \sqrt{s}$ using TP, what is the corresponding $95 \%$ CL search reach for graviton exchange obtainable with TP? For this type of analysis we assume that the $N^{-1} d A / d z$ distributions for each final state fermion are given by their SM values and ask at what value of $M_{H}$ the corresponding ones with graviton exchange become indistinguishable from these. Again we find that above very modest integrated luminosities the errors are completely dominated by systematics; we thus expect our results to again be on the high 


\begin{tabular}{|c|c|}
\hline$E_{C M}(\mathrm{GeV})$ & Reach $(\mathrm{TeV})$ \\
\hline \hline 500 & 5.4 \\
\hline 800 & 8.8 \\
\hline 1000 & 11.1 \\
\hline 1200 & 13.3 \\
\hline 1500 & 16.7 \\
\hline \hline
\end{tabular}

Table 1: Identification reach for $M_{H}$ in the ADD model assuming the distribution $N^{-1} d A / d z \sim 1-z^{2}$ and varying the individual normalizations for the final states $f=\mu, \tau$, $f=b$ and $f=c$ for LC of different center of mass energies.

side of what would be obtained in a more detailed detector study. These results are shown in Table 2 where we see that the values are in the range $M_{H} \gtrsim 20 \sqrt{s}$. These are such enormous numbers that even a degradation by $30-40 \%$ would lead to the highest search reaches for KK graviton exchange found so far in the literature[18].

\begin{tabular}{|c|c|}
\hline$E_{C M}(\mathrm{GeV})$ & Reach $(\mathrm{TeV})$ \\
\hline \hline 500 & 10.2 \\
\hline 800 & 17.0 \\
\hline 1000 & 21.5 \\
\hline 1200 & 26.0 \\
\hline 1500 & 32.7 \\
\hline \hline
\end{tabular}

Table 2: 95\% CL search reach for $M_{H}$ as described in the text.

Given the great sensitivity of transverse polarization to KK graviton/spin-2 exchange it would be natural to ask if TP can be used to distinguish the ADD from the RS model scenarios below KK production threshold. At first, there would seem to be no difference between the predictions of these two models for the situation under discussion. In the RS 
model, if we are away from the $Z$ and graviton KK poles the imaginary part of amplitude which enters the term proportional to $\sin 2 \phi$ becomes vanishingly small. However, as was recently pointed out by Datta, Gabrielli and Mele[19], the exchange of an essentially continuous spectrum of ADD gravitons leads to a finite, cutoff-independent imaginary part of the amplitude. This forgotten piece grows very rapidly with increasing $\sqrt{s}$ and depends quite sensitively upon the number of extra dimensions. Since this term is finite it directly probes the effective fundamental Planck scale of the extra-dimensional theory. Using the notation employed above one now finds that $f_{g}$ has an grown imaginary part:

$$
f_{g}=\frac{\lambda s^{2}}{4 \pi \alpha M_{H}^{4}}\left[1-i \frac{\pi M_{H}^{4}(\sqrt{s})^{\delta-2} S_{\delta-1}}{16 M_{D}^{\delta+2}}\right]
$$

where $\delta$ is the number of extra dimensions, $M_{D}$ is the $\delta$ dimensional fundamental scale and $S_{\delta-1}$ is the area of the $\delta$ sphere. We again note that the magnitude of this new imaginary part, unlike the real part as parameterized in the Hewett scheme, depends quite strongly on the number of extra dimensions.

To proceed we can form a new asymmetry in analogy to the above:

$$
\frac{1}{N} \frac{d A_{i}}{d z}=\left[\frac{\int_{+} \frac{d \sigma}{d z d \phi}-\int_{-} \frac{d \sigma}{d z d \phi}}{\int d \sigma}\right]
$$

where now the $\int_{ \pm}$are integrations over regions where $\sin 2 \phi$ takes on \pm values and we integrate over all $z$ and $\phi$ in the denominator as before. We note that when we perform the integrations in this manner all terms proportional to $\cos 2 \phi$ are found to cancel implying that there is no cross contamination from this other asymmetry source. (This also implies that all of our analyses above will go through even if a term proportional to $\sin 2 \phi$ is present.) Of course this new distribution is identically zero in both the SM as well as the RS model away from the $Z$ and RS KK graviton poles. Thus, observing any non-zero value for this 


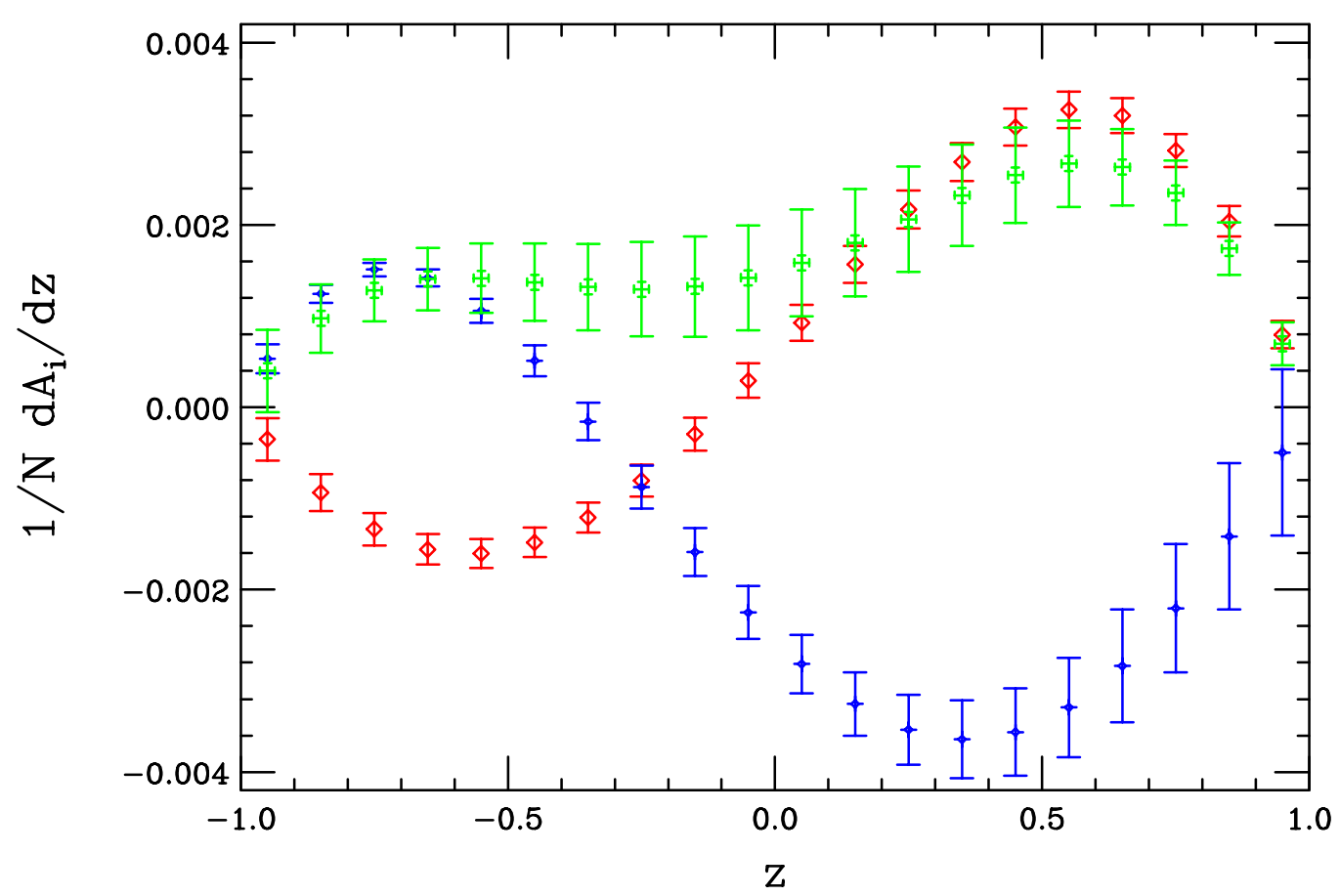

Figure 4: The $N^{-1} d A_{i} / d z$ distributions at a $500 \mathrm{GeV}$ collider assuming $M_{H}=M_{D}=1.5$ $\mathrm{TeV}$ and $\delta=3$ with an integrated luminosity of $500 \mathrm{fb}^{-1}$. The plotted points from top to bottom in the center of the plot correspond to $f=b, \mu$ plus $\tau$ and $c$, respectively.

quantity is a signal for the ADD model. This is particularly true after the spin-2 nature of the exchange has already been established. Fig 4 shows how these new asymmetry distributions may appear at a $500 \mathrm{GeV} \mathrm{LC} \mathrm{assuming} \mathrm{as} \mathrm{before} \mathrm{that} P_{T}=0.8$ and $P_{T}^{\prime}=0.6$ and taking $\delta=3$ for purposes of demonstration. (We do not consider the case $\delta=2$ here as the bounds on the scale $M_{D}$ in this case are in excess of $\left.\sim 100 \mathrm{TeV}[18]\right)$. For simplicity we have assumed $M_{H}=M_{D}$ in this figure and will continue to do so in our discussion below; we expect these two mass scales to be reasonably comparable, though if for some reason $M_{H}<<M_{D}$ this would lead to a serious modification in the sensitivity to this observable.

Assuming a value of $\delta$ we can ask up to what value of $M_{H}=M_{D}$ we can determine that the $N^{-1} d A_{i} / d z$ distribution is non-zero at the $5 \sigma$ level. Based on the expression above we expect that this reach will be reasonably sensitive to the value of $\delta$; this is indeed what we 

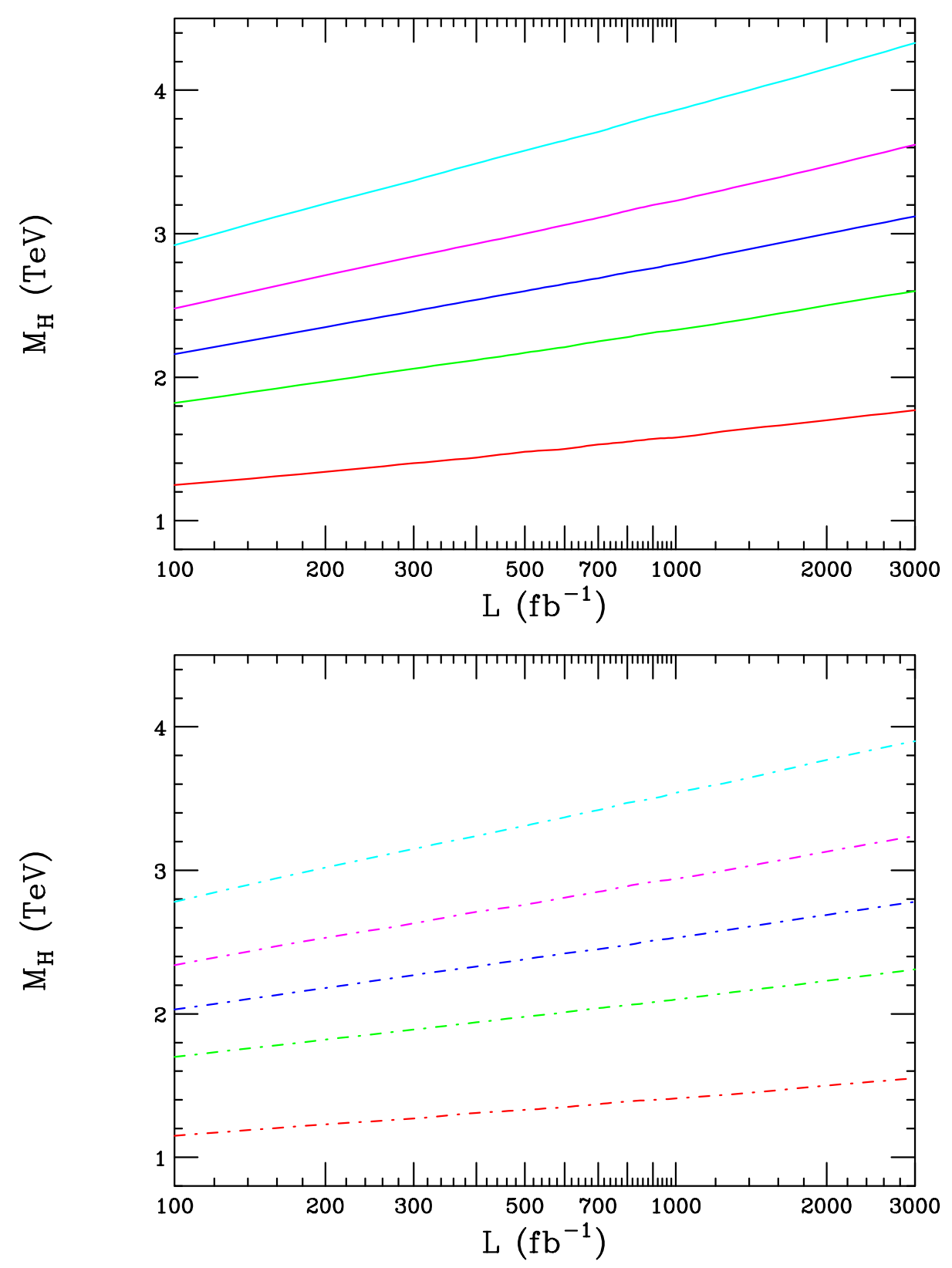

Figure 5: $5 \sigma$ reach for the discovery of a nonzero value of the azimuthal asymmetry $N^{-1} d A_{i} / d z$ distribution as a function of the integrated luminosity at a LC. The top(bottom) panel is for $\delta=3(4)$. Within each panel, from bottom to top the curves are for $\sqrt{s}=0.5,0.8,1,1.2$ and $1.5 \mathrm{TeV}$, respectively. $M_{H}=M_{D}$ is assumed throughout as is $P_{T}=0.8$ and $P_{T}^{\prime}=0.6$. 
find from Figs. 5-6 which show the resulting reaches at the $5 \sigma$ level for the range $\delta=3-6$. The first thing to notice is that the sensitivity to this imaginary term decreases as $\delta$ is increased. This is not surprising since we see from the equation above that the magnitude of this term goes as $\left(\sqrt{s} / M_{H}\right)^{\delta-2}$ and $\sqrt{s} / M_{H}$ is always $<1$ while $\delta \geq 3$. For $\delta=3$ the reach is found to be $\sim(2.5-3) \sqrt{s}$ while for $\delta=6$ one obtains only $\sim 2 \sqrt{s}$. Although these numbers are not large in comparison to those we've obtained in the other analyses above they provide the first indication that these two scenarios can be distinguished at a collider via indirect measurements.

\section{Summary and Conclusion}

Historically, transverse polarization has not received much attention in the literature as a probe for new physics signatures. However, in searching for and identifying new physics at colliders one must make use of as many tools as possible.

In this paper we have examined the possible uses of transverse polarization in searching for, discovering and identifying spin-2 graviton exchange signatures in models with extra dimensions. The results of our analysis are as follows: $(i)$ We have found that the interference of SM and spin-2 graviton KK exchanges leads to contributions to the azimuthal asymmetry distributions which are odd in $\cos \theta$, a rather unique signature. The appearance of such odd terms does not happen in the case of other new physics such as a $Z^{\prime}$, contact interactions, gauge boson KK excitations or the exchange of new scalars. (ii) Using two different sets of observables that probe the integrated contributions of these odd terms, we showed that it possible to differentiate KK graviton/spin-2 exchanges from all other new physics contributions to contact interactions at the $5 \sigma$ level up to ADD cutoff scales of $M_{H} \sim(3.5-4) \sqrt{s}$. (iii) Fitting to the shape of the full differential distribution itself was 

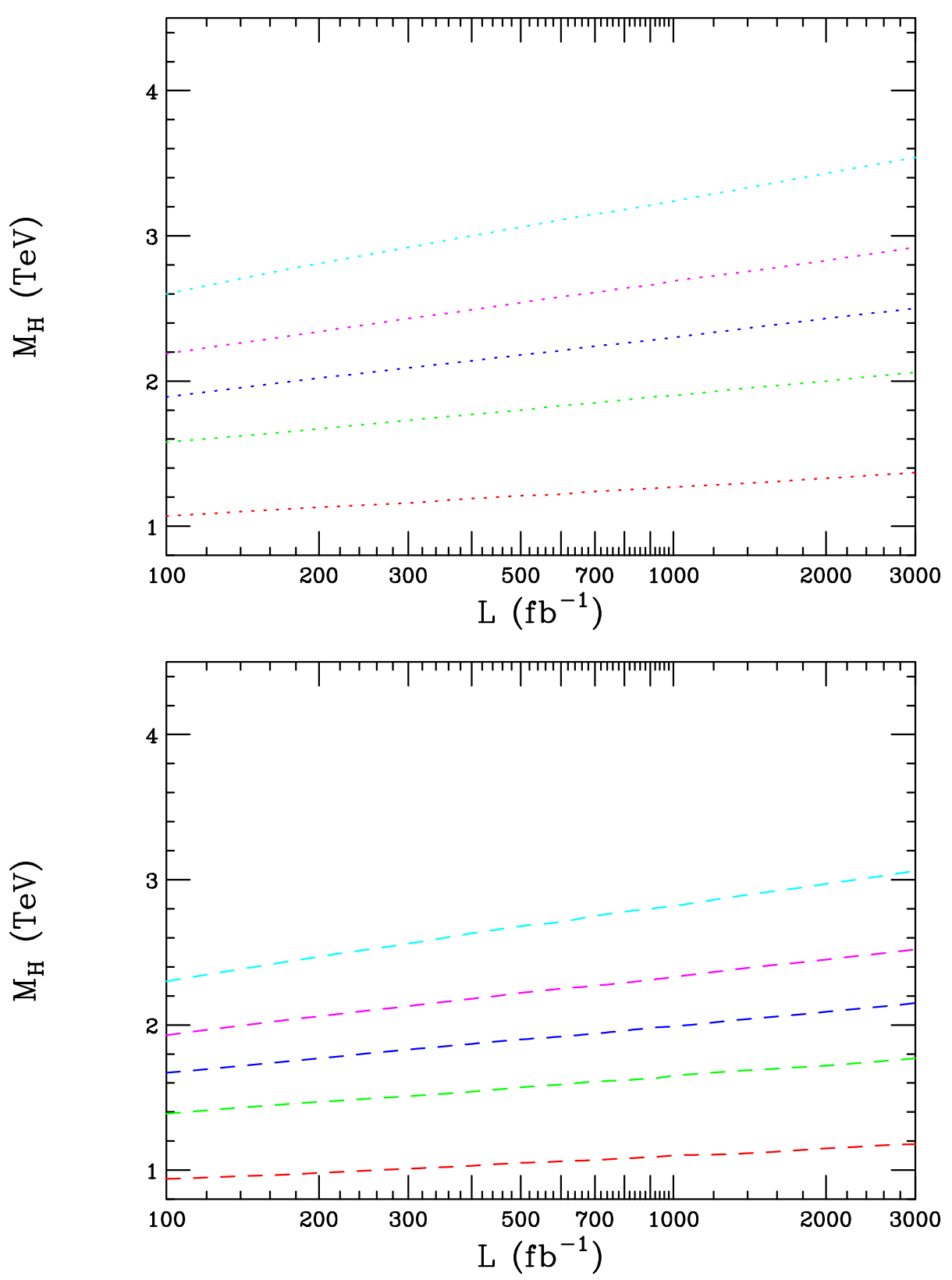

Figure 6: Same as the previous figure but now for $\delta=5$ (top) and $\delta=6$ (bottom). 
shown to provide much more discriminating power; we found that the $5 \sigma$ identification reach was substantially increased to $M_{H}=(10-11) \sqrt{s}$, about a factor two improvement over what we obtained in our earlier analysis in the case of longitudinal polarization. This result is, however, quite sensitive to our assumptions about the sizes of various systematic errors. (iv) Using this same type of analysis we obtained a 95\% CL search reach for new physics in excess of $m_{H}=20 \sqrt{s}$; this is again about a factor of two improvement over other analyses. As in the previous analysis, this result is also quite sensitive to the assumed values of the systematic errors. Clearly, more detailed studies are required to verify these results. $(v)$ In the case of the ADD model, an additional imaginary piece of the amplitude is present in comparison to the RS model below KK production threshold. We showed that this leads to a new asymmetry, produced through transverse polarization, which allows RS and ADD model separation at $5 \sigma$ up to masses $M_{H}=(2.5-3) \sqrt{s}$.

It is clear from our analysis that transverse polarization, though somewhat long neglected, can be a very powerful tool in identifying new physics, particularly in the case of extra dimensions. Further detailed study of the effects examined here may prove extremely useful for future linear collider experiments.

\section{Acknowledgements}

The author would like to thank J.L. Hewett for discussion related to this work. The author would also like to thank K. Desch for an inspirational talk on transverse polarization at the Jeju meeting that returned his interest to this subject after many years.

\section{References}

[1] J. L. Hewett and T. G. Rizzo, Phys. Rept. 183, 193 (1989). 
[2] A. Leike, Phys. Rept. 317, 143 (1999) [arXiv:hep-ph/9805494]; M. Cvetic and S. Godfrey, arXiv:hep-ph/9504216.

[3] W. Buchmuller, R. Ruckl and D. Wyler, Phys. Lett. B 191, 442 (1987) [Erratumibid. B 448, 320 (1999)]; J. L. Hewett and T. G. Rizzo, Phys. Rev. D 58, 055005 (1998) [arXiv:hep-ph/9708419], Phys. Rev. D 56, 5709 (1997) [arXiv:hep-ph/9703337] and Phys. Rev. D 36, 3367 (1987).

[4] J. Kalinowski, R. Ruckl, H. Spiesberger and P. M. Zerwas, Phys. Lett. B 414, 297 (1997) [arXiv:hep-ph/9708272]; J. Kalinowski, R. Ruckl, H. Spiesberger and P. M. Zerwas, Phys. Lett. B 406, 314 (1997) [arXiv:hep-ph/9703436]; T. G. Rizzo, Phys. Rev. D 59, 113004 (1999) [arXiv:hep-ph/9811440].

[5] F. Cuypers and S. Davidson, Eur. Phys. J. C 2, 503 (1998) [arXiv:hep-ph/9609487].

[6] J. L. Hewett, Phys. Rev. Lett. 82, 4765 (1999) [arXiv:hep-ph/9811356]; G. F. Giudice, R. Rattazzi and J. D. Wells, Nucl. Phys. B 544, 3 (1999) [arXiv:hep-ph/9811291]; T. Han, J. D. Lykken and R. J. Zhang, Phys. Rev. D 59, 105006 (1999) [arXiv:hepph/9811350]; T. G. Rizzo, Phys. Rev. D 59, 115010 (1999) [arXiv:hep-ph/9901209].

[7] For an overview of RS phenomenology, see H. Davoudiasl, J. L. Hewett and T. G. Rizzo, Phys. Rev. D 63, 075004 (2001) [arXiv:hep-ph/0006041]; Phys. Lett. B 473, 43 (2000) [arXiv:hep-ph/9911262]; Phys. Rev. Lett. 84, 2080 (2000) [arXiv:hep-ph/9909255]; J. L. Hewett, F. J. Petriello and T. G. Rizzo, JHEP 0209, 030 (2002) [arXiv:hepph/0203091].

[8] N. Arkani-Hamed, S. Dimopoulos and G. R. Dvali, Phys. Lett. B 429, 263 (1998) [arXiv:hep-ph/9803315]; I. Antoniadis, N. Arkani-Hamed, S. Dimopoulos and 
G. R. Dvali, Phys. Lett. B 436, 257 (1998) [arXiv:hep-ph/9804398]; N. Arkani-Hamed, S. Dimopoulos and G. R. Dvali, Phys. Rev. D 59, 086004 (1999) [arXiv:hep-ph/9807344].

[9] L. Randall and R. Sundrum, Phys. Rev. Lett. 83, 3370 (1999).

[10] See, for example, I. Antoniadis, Phys. Lett. B 246, 377 (1990); T. G. Rizzo and J. D. Wells, Phys. Rev. D 61, 016007 (2000) [arXiv:hep-ph/9906234]; M. Masip and A. Pomarol, Phys. Rev. D 60, 096005 (1999) [arXiv:hep-ph/9902467]; T. G. Rizzo, Phys. Rev. D 64, 015003 (2001) [arXiv:hep-ph/0101278].

[11] S. Cullen, M. Perelstein and M. E. Peskin, Phys. Rev. D 62, 055012 (2000) [arXiv:hep$\mathrm{ph} / 0001166]$.

[12] G. Pasztor and M. Perelstein, in Proc. of the APS/DPF/DPB Summer Study on the Future of Particle Physics (Snowmass 2001) ed. R. Davidson and C. Quigg, arXiv:hep$\mathrm{ph} / 0111471$.

[13] R. Budny, Phys. Rev. D14, 2969 (1976); H.A. Olsen, P. Osland and I. Overbo, Phys. Lett. B97, 286 (1980); K. Hikasa, Phys. Rev. D33, 3203 (1986); C.P. Burgess and J.A. Robinson, Int. J. Mod. Phys. A6, 2709 (1991); A. Djouadi, F.M. Renard and C. Verzegnassi, Phys. Lett. B241, 260 (1990); F.M. Renard, Z. Phys. C44, 75 (1989); J.L. Hewett and T.G. Rizzo, Z. Phys. C44, 75 (1987) and Z. Phys. C36, 209 (1987); J. Fleischer, K. Kolodziej and F. Jegerlehner, Phys. Rev. D49, 2174 (1994); for a recent discussion of this option at the LC, see K. Desch, talk given at the International Workshop on the Linear Collider, LCWS2002, Jeju Island, Korea, Aug. 2002.

[14] G. Moortgat-Pick, private communication.

[15] T.G. Rizzo, JHEP 02100132002. 
[16] E. Eichten, K. Lane and M. Peskin, Phys. Rev. Lett. 50, 811 (1983).

[17] J. A. Aguilar-Saavedra et al. [ECFA/DESY LC Physics Working Group Collaboration], "TESLA Technical Design Report Part III: Physics at an e+e- Linear Collider," arXiv:hep-ph/0106315; S. Riemann, TESLA Linear Collider Note LC-TH-2001-007'

[18] For a review of the current and anticipated future collider reaches for extra dimensions, see J. Hewett and M. Spiropulu, "Particle physics probes of extra spacetime dimensions," arXiv:hep-ph/0205106.

[19] A. Datta, E. Gabrielli and B. Mele, hep-ph/0210318. 